\title{
LE $75^{\mathrm{E}}$ ANNIVERSAIRE DE L'AMERICAN METEOROLOGICAL SOCIETY
}

Participer à l'Assemblée annuelle de l'American Meteorological Society (AMS) pendant laquelle douze conférences se tiennent en parallèle est déjà, en soi, une expérience étonnante. Si, en plus, cette Assemblée coïncide avec le $75^{\mathrm{e}}$ anniversaire de l'AMS, y être présent devient un privilège. Invité par Richard E. Hallgren, directeur exécutif, j'ai eu la chance d'assister, en janvier dernier, à cet événement.

Mon propos, dans ce court article, n'est pas de faire un commentaire technique sur les sujets traités par tant de brillants orateurs. Je voudrais seulement livrer quelques impressions personnelles.

On peut aisément imaginer les problèmes posés par l'organisation d'une telle manifestation. L'AMS a montré, une nouvelle fois, son savoir-faire et son professionnalisme. Le résultat a été remarquable, puisque plus de 2500 spécialistes étaient présents.

L'ensemble des conférences, les posters et l'exposition étaient abrités dans un grand hôtel de Dallas (Texas), conçu pour de telles conventions. Avec ses patios intérieurs couverts, il permettait une circulation sans problème entre les différentes sessions. Les salles, modulables, avaient été bien ajustées au nombre prévu de participants dans chaque spécialité. Une seule fois, à ma connaissance,

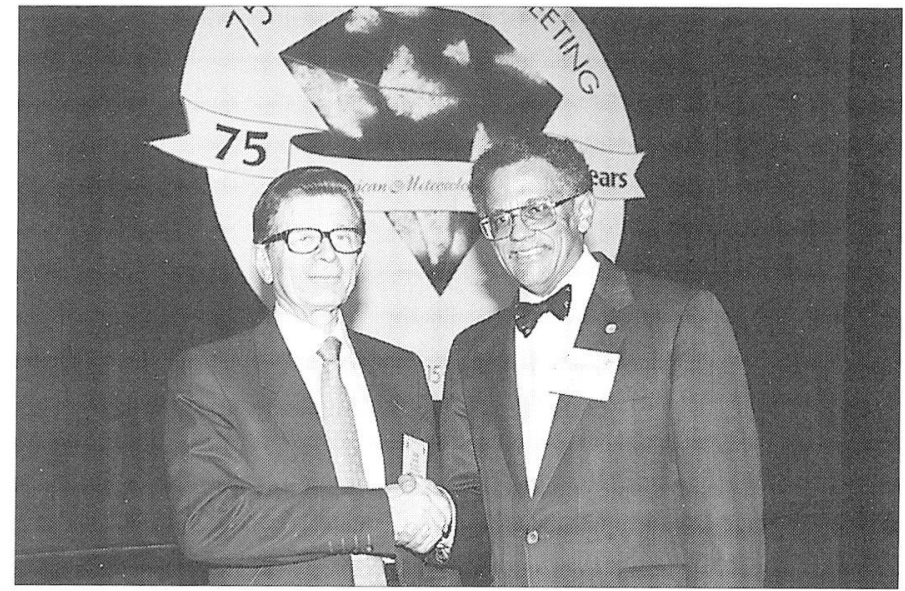

René Morin (président de la SMF, à gauche) et Warren M. Washington (AMS past-president, à droite) pendant le « Diamond Anniversary Awards banquet » à Dallas, États-Unis, à l'occasion du $75^{\circ}$ anniversaire de I'AMS (janvier 1995). cette adéquation a été mise en défaut : la conférence sur «L'évolution de la météorologie dynamique », présentée par Edward N. Lorenz ${ }^{(1)}$, a remporté un tel succès qu'une partie de l'auditoire (dont moi-même) était debout et que nombreux étaient ceux qui se pressaient à l'entrée de la salle pour tenter de capter quelques phrases. Pour le professeur Lorenz, une seule salle aurait convenu : la grande salle de bal de l'hôtel !

Ce qui m’a sans doute le plus frappé, c'est 1'atmosphère tout à la fois studieuse, détendue et très amicale que l'AMS a su créer entre ses membres. Il faut sans doute chercher là une des raisons de son succès. Cette atmosphère s'est particulièrement manifestée lors du dîner de gala, qui réunissait environ 2600 convives dans la salle de bal Chantilly de l'hôtel. Au cours de la soirée, de nombreux prix ont été remis à des personnalités qui se sont distinguées par leurs recherches, leurs écrits ou leurs actions dans le domaine des sciences de l'atmosphère, dont le professeur Edward N. Lorenz pour son livre The Essence of Chaos. On sentait dans les remerciements des récipiendaires qu'ils avaient conscience d'appartenir à une grande famille et qu'ils en étaient fiers. Les étudiants en météorologie n'ont pas été oubliés : parmi eux, plusieurs dizaines ont reçu des aides financières d'industriels, d'organismes nationaux et

(1) Edward N. Lorenz est professeur au Centre de météorologie et d'océanographie physique du Massachusetts Institute of Technology (MIT), à Cambridge, Massachusetts, États-Unis ; il est aussi l'auteur célèbre du livre récent The Essence of Chaos. 
de l'AMS. J'avais rencontré quelques-uns d'entre eux lors d'un dîner qui regroupait également les représentants des sociétés donatrices et des personnalités étrangères. La passion qu'ils exprimaient pour les sciences de l'atmosphère m'a impressionné.

De nombreux présidents ou représentants de Sociétés météorologiques étrangères, invités par l'AMS, étaient venus. Nous nous sommes retrouvés au cours d'une réception plus restreinte qui a suivi le dîner de gala. Ce fut l'occasion d'échanger des informations sur nos projets respectifs, et aussi de féliciter et de remercier l'état-major de l'AMS.

Pendant ces journées, j'ai eu l'occasion de rencontrer à plusieurs reprises les cinq représentants de Météo-France qui présentaient des conférences : Patrick Bénichou, Antoine Lasserre-Bigorry, Stéphane Sénési, Jean-Louis Brenguier et Régis Juvanon du Vachat. Deux d'entre eux m'ont assisté lors de mon entrevue avec Richard E. Hallgren et Kenneth C. Spengler, respectivement directeur exécutif et directeur exécutif emeritus de l'AMS. L'objet de cette réunion était d'examiner les modalités d'une coopération entre nos deux Sociétés, comportant notamment l'organisation en commun de conférences scientifiques en 1997 , comme nous l'avons fait avec succès en 1991.

L'AMS, nous le savons tous, nous donne un grand exemple d'efficacité. Certes, avec ses 11000 membres, elle a des moyens considérables. De plus, son environnement aux États-Unis est très différent du nôtre. Il n'en reste pas moins que je la considère comme un modèle pour la SMF. Bien évidemment, nous resterons toujours à une échelle plus modeste. Mais, du moins, laissons-nous entraîner par son sérieux, son dynamisme et son enthousiasme. Sur le terrain de la déontologie et de l'accréditation, où nous nous engageons actuellement, elle peut également nous apporter l'éclairage d'une longue expérience réussie.

Pour commémorer ce $75^{\mathrm{c}}$ anniversaire de l'AMS et « honorer l'action particulièrement remarquable de ses dirigeants dans le développement et la dissémination du savoir dans le domaine des sciences de l'atmosphère et des sciences annexes », la SMF a décidé de remettre la médaille en vermeil d'Urbain Le Verrier au président sortant, Warren M. Washington, au directeur exécutif, Richard E. Hallgren, et au directeur exécutif emeritus, Kenneth C. Spengler.

Nous venons de recevoir de Warren M. Washington et de Richard E. Hallgren deux lettres de remerciement que nous avons souhaité publier in extenso dans leur traduction française.

Cher Monsieur Morin,

Au nom de l'American Meteorological Society (AMS), je tiens à vous remercier pour la magnifique médaille de vermeil Le Verrier. L'AMS est une société relativement jeune en comparaison des 143 ans d'histoire de la Société météorologique de France. La coopération entre les scientifiques de nos deux pays a toujours été et continuera d'être excellente.

L'organisation de cette réunion scientifique d'importance était une entreprise de taille nécessitant une étroite collaboration entre nos membres, les présidents de sessions et d'ateliers, le bureau de l'AMS et ses différents comités. L'excellence professionnelle du personnel des bureaux de Boston et de Washington D.C. a permis de mener à bien les aspects logistiques primordiaux de la réunion, ce qui a conduit de nombreuses aspects logistiques primordiaux de la reunion, ce qui a condula meilleure jamais organisée. Nous sommes tout spécialement heureux que tant de membres et de Sociétés de l'étranger - dont la SMF - aient pu y participer.

Cordialement, 


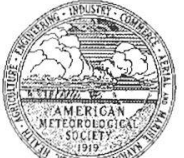

AMERICAN METEOROLOGICAL SOCIETY

45 BEACON STREET, BOSTON, MASSACHUSETTS $02108-3693$ U.S.A.

TEL: $617-227.2425$
FAX: 617-742-8718
INTER INET: halgren@ aip.org

26 April 1995
RICHARD E. HALLGREN, EXECUTIVE DIRECTOR
KENNETH C. SPENGLER, EXECUTIVE DIRECTOR EMTRITU

Dr. René Morin, President

Societe Meteorologique de France

2, avenue Rapp

75340 Paris Cedex 07

Dear René:

All of us at the American Meteorological Society were extremely pleased to receive the Le of the fact that fom the Societe Meteorologique de France. It was very, very special in light several years aon and in Dallas this year are very special to nature and the good friendly humor that underpin the discussions betwoy both the substantive

continue to work on the next meeting in France and hope to be able to respond to you in the next couple of months on that topic. I am very optimistic that it will work out quite well a greement among the people involved here in I want to make sure that I have good

I am really pleased that you could come to the Dallas meeting. I hope you found it very rewarding. I look forward to seeing you in the not too distant future. The Le Verrier medal will always be very special to me and of course to the AMS.

Best regards,

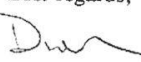

\section{Richard E. Hallgren}

Executive Director

jma

\section{Le 26 avril 1995}

Cher René,

À l'American Meteorological Society, nous avons tous été très heureux de recevoir la médaille Le Verrier de la Société météorologique de France. Elle représente pour nous un honneur qui nous touche tout particulièrement étant donné que vous en êtes actuellement le président. Nos conversations, à Paris il y a plusieurs années et à Dallas récemment, revêtent à mes yeux un aspect tout particulier. J'apprécie à la fois le côté très sérieux et la bonne humeur amicale qui étayent nos discussions.

Je continue de travailler sur la prochaine conférence en France et espère être à même de vous répondre à ce sujet dans les deux prochains mois. Je suis très optimiste quant à la réussite de ce projet, mais avant de faire une déclaration plus définitive, je désire être sûr d'avoir un large accord des personnes concernées ici, aux États-Unis.

Je suis vraiment très heureux que vous ayez pu venir à la réunion de Dallas. J'espère que vous avez trouvé cela gratifiant. J'attends avec impatience de vous rencontrer à nouveau prochainement. La médaille Le Verrier me restera toujours particulièrement chère, ainsi qu'à I'AMS toute entière.

Meilleur souvenir,

Richard E. Hallgren Directeur exécutif American Meteorological Society

Au nom de la SMF, je tiens à remercier une nouvelle fois l'AMS pour son invitation à participer aux cérémonies de son $75^{\circ}$ anniversaire et la félicite pour le grand succès de cette manifestation. Nous espérons, dans un avenir proche, organiser en France avec l'AMS une ou deux conférences internationales, montrant ainsi que nos deux Sociétés ont une seule et même ambition : promouvoir la connaissance de l'environnement atmosphérique.

René Morin

Président de la SMF 